\title{
Form-finding of Tensegrity Structures
}

\section{Utilizing a Nonlinear Fletcher-Reeves Conjugate Gradient Method}

Zhao, L

http://hdl.handle.net/10026.1/17757

\subsection{9/rcar52367.2021.9517591}

2021 IEEE International Conference on Real-time Computing and Robotics (RCAR)

IEEE

All content in PEARL is protected by copyright law. Author manuscripts are made available in accordance with publisher policies. Please cite only the published version using the details provided on the item record or document. In the absence of an open licence (e.g. Creative Commons), permissions for further reuse of content should be sought from the publisher or author. 


\title{
Form-finding of Tensegrity Structures Utilizing a Nonlinear Fletcher-Reeves Conjugate Gradient Method*
}

\author{
Liming Zhao ${ }^{1}$, Keping Liu ${ }^{1}$, Chunxu $\mathrm{Li}^{2}$, Long $\mathrm{Jin}^{3}$, Zhongbo Sun ${ }^{1}$
}

\begin{abstract}
In the domain of soft tensegrity robot, the selfequilibrium tensegrity structure is vital for the further analysis of robot's locomotion. Furthermore, form-finding is an important step for finding a self-equilibrium tensegrity structure. In this paper, a conjugate gradient form-finding (CGFF) algorithm is developed and investigated for the form-finding problems of tensegrity systems. Besides, a Fletcher-Reeves conjugate gradient method is employed to solve the nonlinear unconstrained optimization problems which transformed from the form-finding problems. Moreover, the initial conditions of the tensegrity structure such as the axial stiffness and rest lengths of the element have been utilized to explore the configuration details of the self-equilibrium tensegrity system. Eventually, several numerical simulations are provided to verify the accuracy and high-efficiency of the CGFF form-finding algorithm.
\end{abstract}

\section{INTRODUCTION}

The name of tensegrity is presented by architect Fuller, because of its unique features it has been full developed in many fields such as the soft tensegrity robot and body bionics fields [1].

In the process of designing a tesegrity structure, the technique to find a self-equilibrium tensegrity structure which called form-finding is a crucial step in designing tensegrity structures. In the field of form-finding methods, the force destiny approach has been regarded as a valid approach for forming-finding process of a tensegrity structure. An algorithm which based on numerical analysis to find a selfequilibrium structure has been first presented, it can be regarded as the foundation of force destiny approach [2]. In order to find a group of force density coefficients which fit the rank deficiency conditions, an algorithm that utilized eigenvalue analysis and spectral decomposition has been developed in [3]. In [4], a form-finding method which utilized form-finding eigenvalue decomposition method (FDM)

\footnotetext{
*The work is supported in part by the National Natural Science Foundation of China under grants 61873304, and in part by the China Postdoctoral Science Foundation Funded Project under grant 2018M641784 and 2019T120240, and also in part by the Key Science and Technology Projects of Jilin Province, China, grant nos. 20200201291JC.

${ }^{1}$ Zhongbo Sun is with the Department of Control Engineering, Changchun University of Technology, Changchun 130021, PR China corresponding author e-mail: zhongbosun2012@163. com

${ }^{1}$ Keping Liu and Liming Zhao are with the Department of Control Engineering, Changchun University of Technology, Changchun 130021, PR China liukepingeccut.edu.cn, zhaoliming904035@163. com

${ }^{2}$ Chunxu Li is with Centre for Robotics and Neural Systems, University of Plymouth, Plymouth PL48AA, UK chunxu. 1 i aplymouth.ac.uk

${ }^{3}$ Long Jin is with School of Information Science and Engineering, Lanzhou University, Lanzhou 730000, PR China jinlong@lzu. edu. cn
}

to dispose the form-finding problems has been presented. Moreover, in order to deal with complex tensegrity structures, the Monte Carlo iteration has been utilized to design large scale tensegrity structures with random initial configurations [5]. Besides, a numerical approach by calculating feasible force density vector of the structure has been proposed in [6]. Moreover, several approaches have been developed which utilizing genetic algorithm and two-time singular value decomposition (SVD) method have been proposed to solve the problems of force density coefficients [7] [10]. Moreover, in [11]-[16], the form-finding problem is regarded as an optimization problem. Though these formfinding methods utilized few initial conditions, thus simplifying the form-finding process. However these form-finding algorithms don't involve configuration details of tensegrity systems. In general, the force destiny approach is not relate to configuration details of the tensegrity structure. Therefore, it is worth discussing the configuration details of the tensegrity structures in further research.

Conjugate gradient algorithm has advantages of fast convergence and simple algorithm structure. In [17]-[20], the authors have developed a series of modified conjugate gradient approaches, which show global convergence on dealing nonlinear optimization problems. In [21], a modified projective Dai-Yuan conjugate gradient approach has been proposed to solve the nonlinear optimization problem which converted robust control problem into nonlinear optimization problem by online solving exponential stabilizing robust controller. Besides, the authors also developed a zeroing neural network to solve the time varying nonlinear optimization problems [22]. Therefore, in this paper, a classical conjugate gradient algorithm has been utilized to establish a CGFF method. The CGFF approach has been utilized to solve the nonlinear unconstrained optimization problem which converted from the force destiny linear equilibrium equations. Not only that, the physical informations which include cross sectional area, Young's moduli and rest length are considered to explore the configuration details of the tensegrity system. This paper is organized as follows: In Section II, the nonlinear equilibrium equations modeling process is presented. Besides, the nonlinear unconstrained optimization problem which transformed from the formfinding problem is solved by CGFF method in section III. Furthermore, in section IV, numerical simulations which include two two-dimensional tensegrity structures and a threedimensional tensegrity structure are provided to verify the accuracy of CGFF algorithm. Eventually, Section V describes the conclusion and prospects the future works. 


\section{PROBLEM FORMATIONS}

\section{A. Equation Form of Force Density}

As for a tensegrity structure, it has $v$ elements and $n$ free points, respectively. A topological matrix $\boldsymbol{\rho}$ could represent the topological structure of the presented tensegrity system. The $v$ element, which connect point $i$ and point $j$, can be defined by $d_{i i}=\sqrt{\left(x_{i}-x_{i}\right)^{2}+\left(y_{i}-y_{i}\right)^{2}+\left(z_{i}-z_{i}\right)^{2}}$

$$
\boldsymbol{\rho}=\left\{\begin{array}{cc}
1 & p=i \\
-1 & p=j \\
0 & \text { otherwise }
\end{array}\right.
$$

supposing $i$ and $j$ represent the beginning and end nodal points of the element $v, f_{i, j}$ and $l_{i, j}$ mean the internal force and present length of element $v$. Hence, the force destiny coefficient of element $v$ could be defined as follows:

$$
q_{i, j}=\frac{f_{i, j}}{l_{i, j}}=\frac{l_{i, j}-l_{i, j}^{0}}{l_{i, j}} \times E_{i, j} \times A_{i, j} .
$$

Here, $\mathbf{A}, \mathbf{E}$ and $\mathbf{l}^{\mathbf{0}}$ denote cross sectional area, Young's moduli and rest length, respectively. Assuming that there is a three-dimensional $(d=3)$ tensegrity structure, $\mathbf{x}, \mathbf{y}, \mathbf{z}$ represent nodal coordinates along three directions and $\mathbf{q}$ is the force destiny vector. Therefore, the linear equilibrium equations along three directions can be defined by

$$
\mathbf{U q}=\left[\begin{array}{c}
\boldsymbol{\rho}^{\mathrm{T}} \operatorname{diag}(\boldsymbol{\rho} \mathbf{x}) \\
\boldsymbol{\rho}^{\mathrm{T}} \operatorname{diag}(\boldsymbol{\rho} \mathbf{y}) \\
\boldsymbol{\rho}^{\mathrm{T}} \operatorname{diag}(\boldsymbol{\rho} \mathbf{z})
\end{array}\right] \mathbf{q}=\left[\begin{array}{c}
\mathbf{F}_{\mathbf{x}} \\
\mathbf{F}_{\mathbf{y}} \\
\mathbf{F}_{\mathbf{z}}
\end{array}\right],
$$

which $\mathbf{U}$ is the equilibrium matrix represents the connection between the nodal coordinates and force destiny coefficient matrix. $\mathbf{F}$ is the external force vector.

\section{B. Requirement on Rank Deficiency Conditions}

As for a self-equilibrium tensegrity structure, the force destiny matrix $\mathbf{D}$ can be defined by

$$
\mathbf{D}=\boldsymbol{\rho}^{\mathrm{T}} \operatorname{diag}(\mathbf{q}) \boldsymbol{\rho}
$$

where $\boldsymbol{\rho}$ is topological matrix. So, the null space of $\mathbf{D}$ is defined by

$$
\mathrm{n}_{\mathbf{D}}=\mathrm{n}-\mathrm{r}_{\mathbf{D}}
$$

where $r_{\mathbf{D}}$ is the rank of matrix $\mathbf{D}$, the minimum value of $r_{\mathbf{D}}$ is lager than $d$. Meanwhile, the $\mathrm{n}_{\mathbf{U}}$ means the null space of matrix $\mathbf{U}$, which defined as

$$
\mathrm{n}_{\mathbf{U}}=v-\mathrm{r}_{\mathbf{U}},
$$

the minimum value of $\mathrm{n}_{\mathbf{U}}$ is $\mathrm{n}_{\mathbf{U}} \geq 1$.

\section{Nonlinear Equilibrium Equations}

In this subsection, the cross sectional area, Young's moduli, rest length and nodal coordinates are substituted into the linear equilibrium equations and transformed the linear equilibrium equations into nonlinear form which the coordinates vector $\mathbf{x}, \mathbf{y}$ and $\mathbf{z}$ are the variables.

The connection between the element length and nodal coordinates through topological matrix $\boldsymbol{\rho}$ can be expressed as follow:

$$
\mathbf{l}=\sqrt{(\boldsymbol{\rho} \mathbf{x})^{2}+(\boldsymbol{\rho} \mathbf{y})^{2}+(\boldsymbol{\rho} \mathbf{z})^{2}} .
$$

Substituted (2), (7) into linear equilibrium equations (3), such that the linear equilibrium equations (3) has been transformed into following form:

$$
\mathbf{U} \frac{\left(\sqrt{(\boldsymbol{\rho} \mathbf{x})^{2}+(\boldsymbol{\rho} \mathbf{y})^{2}+(\boldsymbol{\rho} \mathbf{z})^{2}}-\mathbf{l}_{\mathbf{0}}\right) \mathbf{E A}}{\sqrt{(\boldsymbol{\rho} \mathbf{x})^{2}+(\boldsymbol{\rho} \mathbf{y})^{2}+(\rho \mathbf{z})^{2}}}=\left[\begin{array}{c}
\mathbf{F}_{\mathbf{x}} \\
\mathbf{F}_{\mathbf{y}} \\
\mathbf{F}_{\mathbf{z}}
\end{array}\right] .
$$

In the general form-finding process, the tensegrity structure is always in a self-equilibrium situation, which means that there is no external force acting on the tensegrity system. Therefore, in the modeling process, external force vector is equal to zero. Thereby, nonlinear equilibrium equations with external force (8) could be transformed into following form:

$$
-\mathbf{U} \frac{\left(\sqrt{(\rho \mathbf{x})^{2}+(\rho \mathbf{y})^{2}+(\rho \mathbf{z})^{2}}-\mathbf{l}_{\mathbf{0}}\right) \mathbf{E A}}{\sqrt{(\boldsymbol{\rho} \mathbf{x})^{2}+(\rho \mathbf{y})^{2}+(\rho \mathbf{z})^{2}}}=\boldsymbol{\eta}(\mathbf{X}),
$$

where $\boldsymbol{\eta}(\mathbf{X}) \in \mathbb{R}^{3 n}$ represents the external force minus internal force which is the residual force form of the nonlinear equilibrium equations, and $\mathbf{X}=[\mathbf{x}, \mathbf{y}, \mathbf{z}]^{\mathrm{T}} \in \mathbb{R}^{3 \mathbf{n}}$ denotes the nodal coordinates vector. Thus, a relationship which connected the force destiny method and nonlinear equations has been build.

The nonlinear equilibrium equations (9) which transformed from the equilibrium matrix $\mathbf{U}$ and force vector $\mathbf{q}$ could be seen as a nonlinear unconstrained optimization problem. In next section, the Fletcher-Reeves conjugate gradient method is utilized to solve the proposed nonlinear unconstrained optimization problem.

\section{NONLINEAR UNCONSTRAINED OPTIMIZATION PROBLEM}

\section{A. Nonlinear Optimization Problem}

The nonlinear equilibrium equation can not be directly solved by utilizing traditional algorithm because of its highly nonlinear. Moreover, it exists the solution of the equation may cause zero lengths which may lead the whole tensegrity system to instability. Hence, the nonlinear equilibrium equations have been transformed into least-square form. With this approach, the conjugate gradient method has been utilized to solve the nonlinear unconstrained optimization problems which transformed from nonlinear equilibrium equations. In addition, after the nonlinear equilibrium equations are transformed into a nonlinear unconstrained optimization problem through the least square method, the objective function must be a convex function. Hence, the nonlinear equilibrium equations (9) are converted to following form:

$$
\min \mathbf{f}(\mathbf{X})=\frac{1}{2} \sum_{i=1}^{3 n}\left(\boldsymbol{\eta}_{i}^{2}(\mathbf{X})\right) .
$$

Here, $\mathbf{f}(\mathbf{X})$ is equal to $\frac{1}{2}\|\boldsymbol{\eta}(\mathbf{X})\|_{2}^{2}$. The Jacobian matrix of $\boldsymbol{\eta}$ can be exploited to express the derivative of $\mathbf{f}(\mathbf{X})$. The 
Jacobian matrix of $\boldsymbol{\eta}$ could be represented through a firstorder derivative of $\mathbf{H}(\mathbf{X})$ which is defined as follows:

$$
\mathbf{H}(\mathbf{X})=\left[\frac{\partial \boldsymbol{\eta}_{j}}{\partial \mathbf{X}_{i}}\right]_{i=j=1 \ldots 3 n} .
$$

Defining $\mathbf{H}(\mathbf{X})$ as $\nabla \boldsymbol{\eta}\left(\mathbf{X}_{j}\right)$, then

$$
\nabla \mathbf{f}(\mathbf{X})=\mathbf{H}(\mathbf{X})^{\mathrm{T}} \boldsymbol{\eta}(\mathbf{X})=\sum_{j=1}^{3 n} \nabla \boldsymbol{\eta}_{j}(\mathbf{X}) \boldsymbol{\eta}(\mathbf{X}) .
$$

It's worth noting that $\nabla \mathbf{f}(\mathbf{X})$ is gradient of $\mathbf{f}(\mathbf{X})$, the conjugate gradient method doesn't need to calculate the Hessian matrix. Thereby, it is a more simpler approach for formfinding of tensegrity structure which could reduce the complexity of calculation.

\section{B. Conjugate Gradient Method}

The traditional way for conjugate gradient method is to update the nodal coordinates as follows:

$$
\mathbf{X}_{k+1}=\mathbf{X}_{k}+\alpha \mathbf{d}_{k},
$$

where $\alpha$ denotes the search step length and $\mathbf{d}_{k}$ means search direction. In general, conjugate gradient method is an optimization algorithm that to generate conjugate direction of Hessian matrix about the minimum point of convex quadratic function by utilizing descent direction at the current point for each iteration step. Besides, evaluating whether the gradient of the objective function $\nabla \mathbf{f}\left(\mathbf{X}_{k}\right)$ is less than the setting error. Yet different from the traditional way, this algorithm works by estimating $\mathbf{d}_{k}$ which is set equal to $\alpha \mathbf{d}_{k}$ whether or not fulfill the setting error. From that, the step length can be updated at any moment during the iterative process. In CGFF algorithm, how to adjust search step $\alpha$ is an important step for the optimization algorithm. If $\alpha$ is too small, the iteration process may cost too much time. On the contrary, if $\alpha$ is too large, the current $\mathbf{d}_{k}$ may not the correct descent direction and may miss the optimal solution. In this paper, a FletcherReeves conjugate gradient form-finding approach has been utilized to solve the nonlinear unconstrained optimization problem which transformed from form-finding problems.

Afterwards, calculating the Jacobian matrix $\mathbf{H}(\mathbf{X})$ and $\nabla \mathbf{f}(\mathbf{X})$ which can be regarded as the gradient of $\mathbf{f}(\mathbf{X})$. Then, $\mathbf{d}_{k}$ has been utilized to update the nodal coordinate $\mathbf{X}$ and the 2-norm of $\mathbf{d}_{k}$ is the cut-off condition of the CGFF method. The current nodal coordinates $\mathbf{X}$ could been seen as the suitable nodal coordinates when the 2-norm of $\mathbf{d}_{k}$ is less than setting error. Otherwise, updating the $\mathbf{f}\left(\mathbf{d}_{k}\right)$ by utilizing current nodal coordinates $\mathbf{X}$ when the 2-norm of $\mathbf{d}_{k}$ is greater than setting error. Ulteriorly, if $\mathbf{f}\left(\mathbf{X}_{k+1}\right)$ is less than $\mathbf{f}\left(\mathbf{X}_{k}\right)$, set $\alpha=\alpha / \beta$ and $k=k+1$, the opposite of this circumstance is when $\mathbf{f}\left(\mathbf{X}_{k+1}\right)$ is greater than $\mathbf{f}\left(\mathbf{X}_{k}\right)$, then setting $\alpha=\alpha \cdot \beta$. Via this approach, the search direction $\mathbf{d}_{k}$ could be adjusted. The algorithm details are shown in Table I and the flowchart of the CGFF approach is shown in Fig. 1.

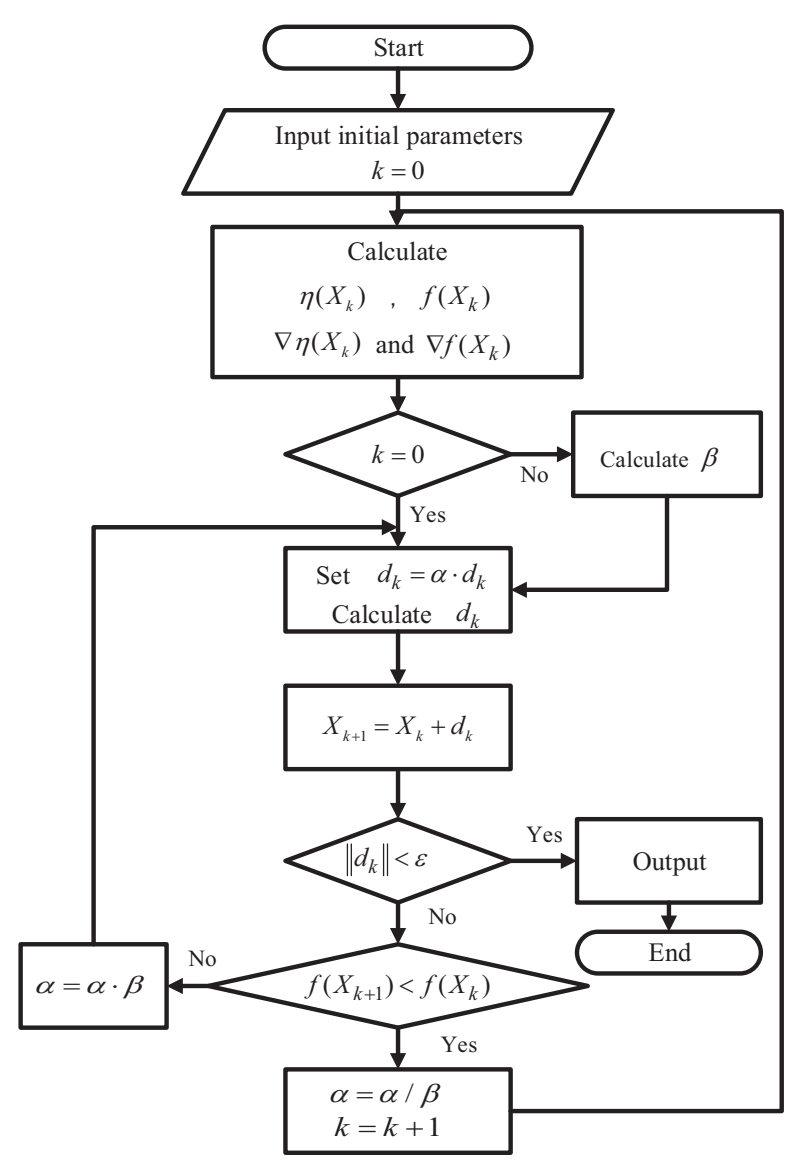

Fig. 1. Flowchart of the CGFF method

\section{NUMERICAL EXPERIMENT}

In this section, the accuracy and high-efficiency of CGFF approach has been proved through three examples including two two-dimensional polygons and a three-dimensional prism.

\section{A. Two-dimensional Tensegrity Structures}

Firstly, a two-dimensional two-strut tensegrity structure which is consisted of 4 cables and 2 struts has been selected to find the self-equilibrium configuration in this subsection. Fig. 2 shows the obtained topological structure of self-equilibrium tensegrity structure. The obtained nodes in Fig. 3 show the different iteration speed under different setting error. As shown in Fig.3, with the improvement of the design error accuracy, the iteration steps are increased. The chosen error is $\varepsilon=1 \times 10^{-8}$ which corresponding to the eighth point and form-finding procedure converges in ten iterations. The obtained nodal coordinates which utilizing CGFF method lying in Table II are as consistent as the obtained nodal coordinates by using FDM approach. The comparison between two series of coordinates can prove the accuracy of the CGFF algorithm. Secondly, in order to verify the CGFF approach still valid in the two-dimensional space, a 
TABLE I

CGFF APPROACH PROCESS

\begin{tabular}{|c||l|}
\hline Steps & CGFF approach details. \\
\hline 2. & Input initial conditions, set $k=0$. \\
& Calculate $\boldsymbol{\eta}\left(\mathbf{X}_{k}\right), \mathbf{f}\left(\mathbf{X}_{k}\right), \nabla \boldsymbol{\eta}\left(\mathbf{X}_{k}\right), \nabla \mathbf{f}\left(\mathbf{X}_{k}\right)$. \\
& If $k$ is equal to 0 \\
& Calculate $\mathbf{d}_{k}, \mathbf{X}_{k+1}$ and $2-\operatorname{norm}\left(\mathbf{d}_{k}\right)$. \\
& While $\left\|\mathbf{d}_{k}\right\|_{2}>\varepsilon$, calculate $\mathbf{f}\left(\mathbf{X}_{k+1}\right)$, \\
& otherwise output $\mathbf{X}$. \\
& If $\mathbf{f}\left(\mathbf{X}_{k+1}\right)<\mathbf{f}\left(\mathbf{X}_{k}\right)$, set $\alpha=\alpha / \beta$ \\
& and $k=k+1$. \\
& Back to calculate $\boldsymbol{\eta}\left(\mathbf{X}_{k}\right), \mathbf{f}\left(\mathbf{X}_{k}\right), \nabla \boldsymbol{\eta}\left(\mathbf{X}_{k}\right)$ \\
& and $\nabla \mathbf{f}\left(\mathbf{X}_{k}\right)$. \\
& Else set $\alpha=\alpha \cdot \beta$ then back to calculate \\
& $\mathbf{d}_{k}, \mathbf{X}_{k+1} \cdot$ \\
& End if \\
& End while \\
& End if \\
If $k$ is not equal to 0 \\
Calculate $\beta, \mathbf{d}_{k}, \mathbf{X}_{k+1}$ and $2-$ norm $\left(\mathbf{d}_{k}\right)$. \\
While $\left\|\mathbf{d}_{k}\right\|_{2}>\boldsymbol{\varepsilon}$, calculate $\mathbf{f}\left(\mathbf{X}_{k+1}\right)$, \\
otherwise output $\mathbf{X}$. \\
If $\mathbf{f}\left(\mathbf{X}_{k+1}\right)<\mathbf{f}\left(\mathbf{X}_{k}\right)$, set $\alpha=\alpha / \beta$ \\
and $k=k+1$. \\
Back to calculate $\boldsymbol{\eta}\left(\mathbf{X}_{k}\right), \mathbf{f}\left(\mathbf{X}_{k}\right), \nabla \boldsymbol{\eta}\left(\mathbf{X}_{k}\right)$ \\
and $\nabla \mathbf{f}\left(\mathbf{X}_{k}\right)$. \\
Else set $\alpha=\alpha \cdot \beta$ then back to calculate \\
$\mathbf{d}_{k}, \mathbf{X}_{k+1} \cdot$ \\
End if \\
End while \\
End if \\
Output the nodal coordinates $\mathbf{X}$
\end{tabular}

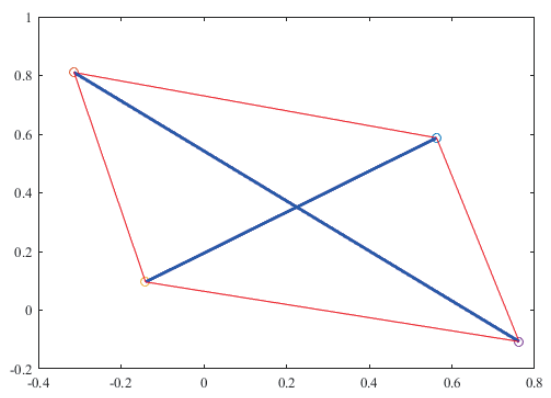

Fig. 2. The obtained geometry of the two-dimensional two-strut tensegrity structure

more complicated self-equilibrium structure is found through the CGFF method for a hexagon tensegrity structure which is formed by three struts and six cables. The self-equilibrium configuration of this tensegrity structure are obtained in Fig.4. The CGFF algorithm converges in ten iterations, and design error is $\varepsilon=1 \times 10^{-8}$ which corresponding to eighth point in Fig.5. The nodal coordinates of hexagon tensegrity structure have shown in Table III. Analogously, the nodal coordinates which obtained by CGFF method are compared with the coordinates obtained by FDM approach, the results are very similar which could also prove the accuracy of CGFF algorithm.

\section{B. Three-dimensional Triangular Prism Tensegrity Structure}

To prove CGFF method is still effective in threedimensional space, a three-dimensional triangular prism

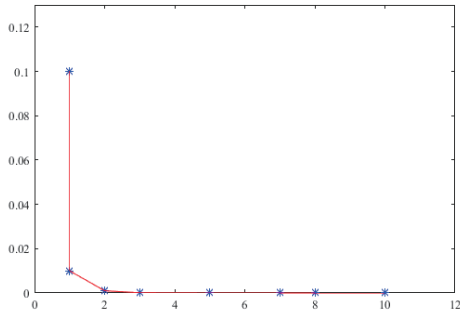

Fig. 3. The convergence of CGFF method for two-dimensional two-strut tensegrity structure

TABLE II

SElF-EQUilibrium NodAl COORDINATES

\begin{tabular}{|c||ccccc|}
\hline \multicolumn{1}{|c||}{ Form-finding method } & \multicolumn{5}{c|}{ Nodal coordinates } \\
\hline \multirow{2}{*}{ CGFF method } & $\mathbf{x}:$ & 0.5345 & -0.2941 & -0.0842 & 0.7737 \\
& $\mathbf{y :}$ & 0.5355 & 0.8044 & 0.1856 & -0.0635 \\
\hline \multirow{2}{*}{ FDM method } & $\mathbf{x}:$ & 0.5366 & -0.2974 & -0.0821 & 0.7716 \\
& $\mathbf{y :}$ & 0.5499 & 0.8094 & 0.1959 & -0.0636 \\
\hline
\end{tabular}

tensegrity structure is presented in this subsection. A triangular prism tensegrity structure is consisted of 3 struts and 9 cables. The self-equilibrium geometry of triangular prism tensegrity structure is shown in Fig.6. The form-finding approach converges in twelve iterations and the design error is $\varepsilon=1 \times 10^{-8}$ which is the eighth point in Fig.7. The nodal coordinates of self equilibrium for triangular prism tensegrity structure have shown in Table IV. Besides, the obtained coordinates by utilizing CGFF algorithm are largely consistent with the nodal coordinates which is obtained by the FDM method. These nodal coordinates which obtained by utilizing two different form-finding methods can prove the accuracy of the CGFF approach in three dimension.

From aforementioned three tensegrity systems formfinding examples, through comparing the nodal coordinates between the CGFF method and FDM approach, the efficient of the CGFF method could be proved. Although the error between two methods is existed, however, the error can be reduced through adjusting $\alpha$ and $\beta$. The current error $\varepsilon=1 \times 10^{-8}$ could satisfy the need of form-finding process for tensegrity structures. If calibrating the parameters $\alpha$ and $\beta$ further, the design error can not change drastically and it

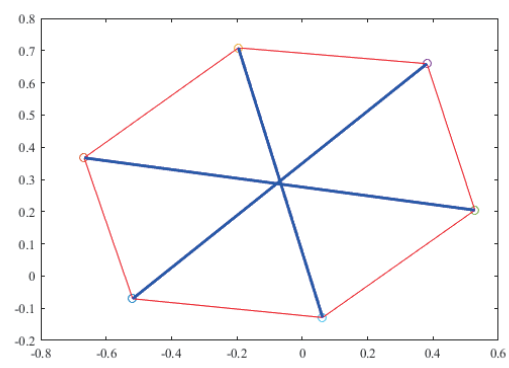

Fig. 4. The obtained geometry of the two-dimensional hexagon tensegrity structure 
TABLE III

SELF-EQUilibrium NodAl COORDINATES

\begin{tabular}{|c||ccccccc|}
\hline \multicolumn{1}{|c|}{ Form-finding method } & \multicolumn{7}{|c|}{ Nodal coordinates } \\
\hline \multirow{2}{*}{ CGFF method } & $\mathbf{x}:$ & -0.4916 & -0.610 & -0.1886 & 0.3527 & 0.4719 & 0.0497 \\
& $\mathbf{y :}$ & -0.0270 & 0.3463 & 0.6659 & 0.6111 & 0.2378 & -0.0812 \\
\hline \multirow{2}{*}{ FDM method } & $\mathbf{x}:$ & -0.5189 & -0.6673 & -0.1955 & 0.381 & 0.5281 & 0.0622 \\
& $\mathbf{y}:$ & -0.0705 & 0.3670 & 0.7086 & 0.6582 & 0.2046 & -0.1288 \\
\hline
\end{tabular}

TABLE IV

SELF-EQUilibrium NODAl COORDINATES

\begin{tabular}{|c||ccccccc|}
\hline Form-finding method & \multicolumn{7}{|c|}{ Nodal coordinates } \\
\hline \multirow{3}{*}{ CGFF method } & $\mathbf{x}:$ & 1.7321 & -0.866 & -0.866 & -1.5 & 0 & 1.5 \\
& $\mathbf{y :}$ & 0 & 1.5 & -1.5 & 0.866 & -1.7321 & 0.866 \\
& $\mathbf{z :}$ & 0 & 0 & 0 & 6.1485 & 6.1485 & 6.1485 \\
\hline \multirow{3}{*}{ FDM method } & $\mathbf{x :}$ & 1.6327 & -0.855 & -0.789 & -1.399 & -0.031 & 1.437 \\
& $\mathbf{y :}$ & 0.038 & 1.3994 & -1.437 & 0.8558 & -1.6358 & 0.789 \\
& $\mathbf{z :}$ & 0.095 & 0.095 & 0.095 & 6.0548 & 6.0548 & 6.0548 \\
\hline
\end{tabular}

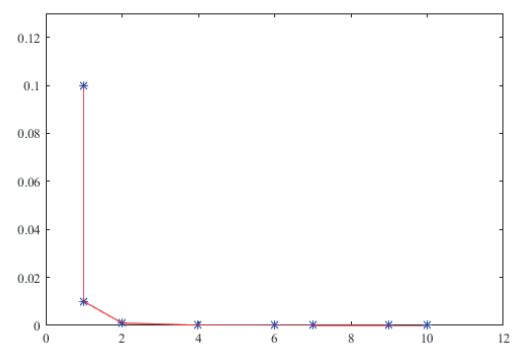

Fig. 5. The convergence of CGFF method for two-dimensional hexagon tensegrity structure

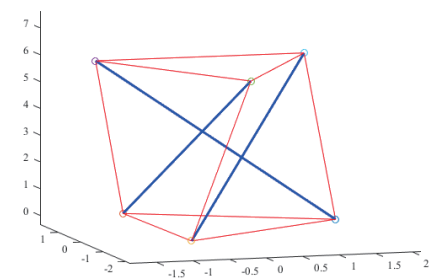

Fig. 6. The obtained geometry of three-dimensional triangular prism tensegrity structure

may cause the non-convergence of form-finding process.

Eventually, as an important index to test the efficiency of the CGFF algorithm, the comparison of computation time between different methods is taken into account. By comparing the computation time of finding the self-equilibrium configuration of two-dimensional hexagon tensegrity structure, the efficiency of the CGFF algorithm could be verified. Utilizing the CGFF approach and the built-in function "fsolve" of MATLAB which could be utilized to solve the nonlinear optimization problems for comparison. The form-finding process of hexagon tensegrity structure which using CGFF method takes 49.76 seconds to find the self-equilibrium structure with the design error $\varepsilon=1 \times 10^{-8}$. In contrast, the built-in function "fsolve" takes 255.08 seconds to find the

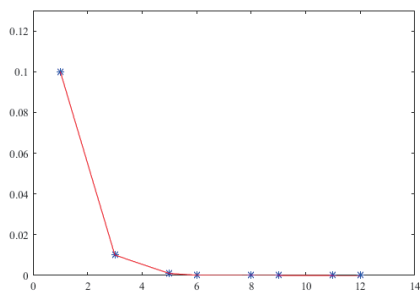

Fig. 7. The convergence of CGFF method for three-dimensional triangular prism tensegrity structure

self-equilibrium configuration of the tensegrity system with the same design error. By means of these results, the highefficiency of the proposed CGFF method could be proved.

\section{CONCLUSIONS}

In this paper, from an optimization algorithm viewpoint, a CGFF approach has been proposed to be utilized during the form-finding process of tensegrity systems. By involving the physical informations of the structure in the nonlinear equilibrium equations, the configuration details of the tensegrity structure could be obtained. Utilizing conjugate gradient approach to solve the nonlinear unconstrained optimization problem which transformed from the linear equilibrium equations. Numerical results have ascertained the accuracy and high-efficiency of the presented CGFF approach for tensegrity structure form-finding solving. Future work will consider the application of neural network algorithms for the form-finding problems of tensegrity structures.

\section{ACKNOWLEDGMENT}

The authors would like to thank the anonymous reviewers and the Technical Editor for their valuable comments and suggestions on revising this paper.

\section{REFERENCES}

[1] K. Kim, A. K. Agogino and A. M. Agogino, Rolling locomotion of cable-driven soft spherical tensegrity robots, Soft Robotics, vol. 7, no. 3, pp. 346-361, Jun. 2020. 
[2] H. J. Schek, The force density method for form finding and computation of general networks, Computer Methods in Applied Mechanics and Engineering, vol. 3, no. 1, pp. 115-134, Jan. 1974.

[3] J. Y. Zhang and M. Ohsaki, Adaptive force density method for formfinding problem of tensegrity structures, International Journal of Solids and Structures, vol. 43, no. 18, pp. 5658-5673, Sep. 2006.

[4] G. G. Estrada, H. J. Bungartz and C. Mohrdieck, Numerical formfinding of tensegrity structures, International Journal of Solids and Structures, vol. 43, no. 22, pp. 6855-6868, Nov. 2006.

[5] Y. Li, X. Q. Feng, Y. P. Cao and H. J. Gao, A Monte Carlo form-finding method for large scale regular and irregular tensegrity structures, International Journal of Solids and Structures, vol. 47, no. 14, pp. 1888-1898, Jul. 2010

[6] H. C. Tran and J. Lee, Form-finding of tensegrity structures with multiple states of self-stress, Acta Mechanica, vol. 222, pp. 131-147, Aug. 2011.

[7] S. Lee and J. Lee, Advanced automatic grouping for form-finding of tensegrity structures, Structural and Multidisciplinary Optimization, vol. 55, pp. 959-968, Aug. 2016.

[8] S. Lee, D. S. Gan and J. Lee, A fully automatic group selection for form-finding process of truncated tetrahedral tensegrity structures via a double-loop genetic algorithm, Composites Part B, vol. 106, pp. 308-315, Dec. 2016.

[9] S. Lee, J. Lee and J. W. Kang, Results of generalized equilibrium path from form-finding of tensegrity structure, International Journal of Steel Structures, vol. 17, pp. 1225-1231, Sep. 2017.

[10] H. C. Tran and J. Lee, Form-finding of tensegrity structures using double singular value decomposition, Engineering with Computers, vol. 29, pp. 71-86, Sep. 2011.

[11] J. G. Cai and J. Feng, Form-finding of tensegrity structures using an optimization method, Engineering Structures, vol. 104, pp. 126-132, Dec. 2015.

[12] X. F. Yuan, S. Ma and S. H. Jiang, Form-finding of tensegrity structures based on the Levenberg-Marquardt method, Computers and Structures, vol. 192, pp. 171-180, Nov. 2017.

[13] J. G. Cai, X. Y. Wang, X. W. Deng and J. Feng, Form-finding method for multi-mode tensegrity structures using extended force density method by grouping elements, Composite Structures, vol. 187, pp. 1-9, Mar. 2018

[14] L. Y. Zhang, S. X. Zhu, S. X. Li and G. K. Xu, Analytical form-finding of tensegrities using determinant of force-density matrix, Composite Structures, vol.189, pp. 87-98, Jan. 2018.

[15] K. Koohestani, On the analytical form-finding of tensegrities, Composite Structures, vol. 166, pp. 114-119, Apr. 2017

[16] S. Ma, X. F. Yuan and S. D. Xie, A new genetic algorithm-based topology optimization method of tensegrity tori, KSCE Journal of Civil Engineering, vol. 23, pp. 2136-2147, Feb. 2019.

[17] Z. B. Sun, Y. T. Tian and H. Y. Li, Two modified three-term type conjugate gradient methods and their global convergence for unconstrained optimization, Mathematical Problems in Engineering vol. 2014, pp. 1-9, Nov. 2014.

[18] Z. B. Sun, H. Y. Li, J. Wang and Y. T. Tian, A gait optimization smoothing penalty function method for bipedal robot via DMOC IFAC-Papers OnLine, vol. 48, no. 28, pp. 1148-1153, Dec. 2015.

[19] Z. B. Sun, Y. T. Tian and J. Wang, A novel projected Fletcher-Reeves conjugate gradient approach for finite-time optimal robust controller of linear constraints optimization problem: application to bipedal walking robots, Optimal Control Applications and Methods, vol. 39, no. 1, pp. 130-159, Jul. 2017.

[20] Z. B. Sun, H. Y. Li, J. Wang and Y. T. Tian, Two modified spectral conjugate gradient methods and their global convergence for unconstrained optimization, International Journal of Computer Mathematics, vol. 95, no. 10, pp. 2082-2099, Aug. 2017.

[21] M. Wang, Z. B. Sun, B. C. Zhang, Z. X. Pang and D. W. Jiang, A Dai-Yuan conjugate gradient algorithm of linear equation constrained optimization approach for optimal robust controller of bipedal robots, International Journal of Advanced Robotic Systems, pp. 1-12, Oct. 2019.

[22] Z. B. Sun, T. Shi, L. Wei, Y. Y. Sun, K. P. Liu and L. Jin, Noisesuppressing zeroing neural network for online solving time-varying nonlinear optimization problem: a control-based approach, Neural Computing and Applications, vol. 32, pp. 11505-11520, 2020. 